\title{
Differential Analysis of Neurodegenerative Aging-Related Mitochondrial Genes of Long-Lived Naked Mole-Rat
}

\author{
Layal Abo Khayal, Ivo Provaznik, and Ewryst Tkacz
}

\begin{abstract}
Analyzing the differential genetic traits related to senescence of the long-lived naked mole-rat and various species that are convergent phylogenetically, by a combination of bioinformatic algorithms with nucleotide genomic signal processing, and hierarchical cluster methods. Since the naked mole-rat has distinctive aging resistance, comparing the aging-related genes may guide to essential differences in pathological incidences of aging diseases among the species concerned.
\end{abstract}

Index Terms-Aging-related genes, DNA damage, neurodegenerative diseases, naked mole-rat (Heterocephalus glaber).

\section{INTRODUCTION}

The naked mole-rat (Heterocephalus glaber) is a unique long-lived organism. It reveals noticeable resistance to chronic diseases of aging. Recently has not been noticed any kind of neoplasms in it. Therefore comparative analysis of Heterocephalus glaber (H. glaber), in parallel with closely-related species of different lifespans, can be used to enhance the understanding of the evolution of longevity, as well as of the molecular, cellular, and genetic mechanisms of aging. It may guide to interpretation of the differences in aging between mammals, and provide major insights into human aging, longevity, and disease susceptibility. Furthermore the sequence of those species may allow the identification of functional elements in the human genome associated with longevity [1].

Differences in maximum longevity of organisms, both in the wild and in the captivity, reflect the differences in disease susceptibility, particularly the susceptibility of aging-related diseases [2]. However the similarity in the sequence of aging-related genes suggests investigation of post-genomic methods such as gene expression [3] and large-scale RNAi screens [4].

Neurodegenerative diseases like Alzheimer's and

Manuscript received October 15, 2012; revised January 2, 2013. This work was supported in part by European Regional Development Fund Project FNUSA-ICRC No. CZ.1.05/1.1.00/02.0123 and by the grant project GACR P102/11/1068 Nano BioTE Cell.

Layal Abo Khayal is with the Brno University of Technology, Faculty of Electrical Engineering and Communication, Department of Biomedical Engineering, Czech Republic (e-mail: khayal@phd.feec.vutbr.cz).

Ivo Provaznik is with the International Clinical Research Center - Center of Biomedical Engineering, St. Anne's University Hospital Brno, Brno, Czech Republic, and with Brno University of Technology, Faculty of Electrical Engineering and Communication, Department of Biomedical Engineering, Czech Republic (e-mail: provaznik@feec.vutbr.cz).

Ewaryst Tkacz is with the Silesian University of Technology, Faculty of Biomedical Engineering and with Brno University of Technology, Faculty of Electrical Engineering and Communication, Department of Biomedical Engineering, Czech Republic (e-mail: tkacz@feec.vutbr.cz).
Parkinson's syndrome and Leber's hereditary optic neuropathy (LHON), are among the major health concerns of aging adults. And since the naked mole-rat shows noticeable resistance to neurodegenerative disease susceptibility and its mitochondrial genes sequences are available, two mitochondrial genes, i.e. COX1 cytochrome c oxidase subunit I and CYTB cytochrome $b$, were chosen for comparative analysis [5], [6], [7].

Mitochondrial Nucleotide Genomic Signals (mtNuGSs) for various species have been analyzed comparatively, since the conversion of nucleotide sequences to Nucleotide Genomic Signals (NuGSs) allows to process them as digital signals [8], [9].

Artificial intelligence $(A I)$ techniques such as symbolic machine learning approaches (nearest-neighbours and identification tree techniques) can be successfully employed in bioinformatics problems, which do not have strong constraints. Whereas AI approaches excel when dealing with problems which do not require 'the absolutely provably correct or best' answer (a 'strong' constraint) but simply require an answer, which is acceptable, within certain defined constraints (a 'weak' constraint) [10].

\section{THE NAKED MOLE- RAT}

The naked mole-rat is the longest-lived rodent with a much longer lifespan than expected for its relatively small body size (see Fig. 1). It is an underground dwelling, eusocial rodent native to north Eastern Africa [11]. H. glaber has a record longevity of approximately 30 years, which makes it the longest-lived subterranean rodent. This subterranean rodent is resistant to neoplasia and it can be used primarily as the first model of resistance to chronic diseases of senescence. Overall, $H$. glaber genes may be the major players in the genetic suppression of tumour formation, and this may open the door for further potential applications in human [12].

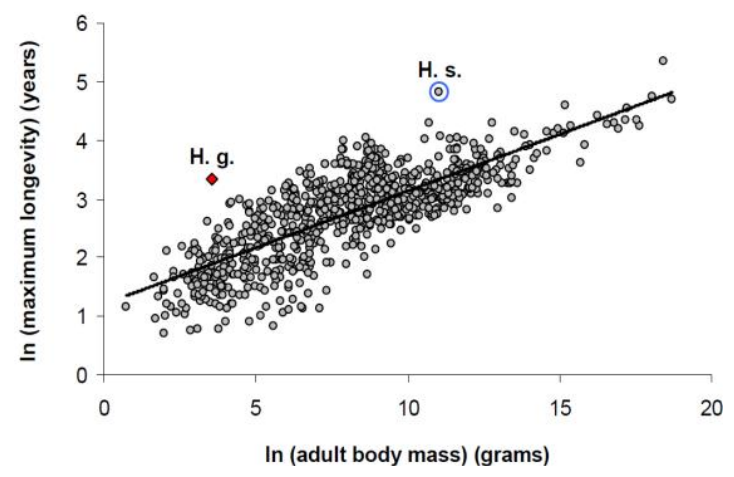

Fig. 1. Plot of the relationship between transformed maximum longevity and typical adult body mass for non-volant mammals $(n=820)$. Heterocephalus glaber (H. g.) is highlighted by a red square. H. s. = Homo sapiens (in blue) [13]. 
H. glaber belongs to the Bathyergidae family [14], [15], which is part of the Hystricognathi suborder of rodents that includes the new world caviomorpha and the old world phiomorpha (see Fig. 2). Fossil evidence suggests that this suborder diverged from the Sciurognathi suborder to which mice and rats belong, about 55 million years ago. Furthermore, these mouse-sized rodents continue to breed well into their third decade of life and show attenuated age-related changes in physiological function [16], [17], such as maintenance of vascular youthfulness into old age [18]. Strikingly, cancer has not been observed to date in $H$. glaber .

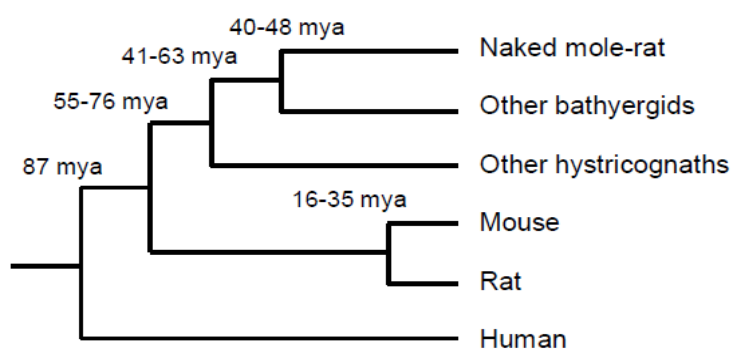

Fig. 2. Phylogeny of Heterocephalus glaber and related species. H. glaber diverged from other bathyergids (i.e., other mole-rats) about 40-48 million of years and from other hystricognaths (e.g., Hystrax and Cavia) about 41-63 mya. Phylogeny from [11], [14], [19], [20].

Aging is widely regarded as the result of accumulating damage [21], and many sources of cellular and molecular damage have been proposed as crucial in aging, oxidative damage, senescent cells, protein toxicity, DNA alterations, and changes in mitochondria. Whereas the accumulation of oxidative damage underlies the process of aging, and these differences between species in reactive oxygen production contribute to differences in aging [22], [23]. Thus the $H$. glaber must have evolved mechanisms to slow the accumulation of different forms of damage.

\section{NuCleotide GENOMIC Signals}

The conversion of nucleotide sequences to Nucleotide Genomic Signals (NuGSs) allows processing them as digital signals, and also makes the straightforward comparison of nucleotide sequences. Fast identification of mutations and inserts, and recognition of the patterns and regularities in the distribution of nucleotide pairs along the sequences are available. The methodology has been proved for the local analysis of the sequences, and for mtDNA analysis as well [25]. The study of pathogen variability and the identification of drug resistance have also been performed [24]. The regularities in the nucleotide distribution (imbalance) and nucleotide pair distribution, follow evoke Chargaff's laws that DNA from any cell of all organisms should have a 1:1 ratio (base Pair Rule) of pyrimidine and purine bases [26]-[8].

The best mapping in revealing the regularities is a one-to-one unbiased complex representation of nucleotide equivalence classes, which attaches complex numbers to adenine, cytosine, guanine and thymine nucleotides:

$$
a=1+j, c=-1-j, g=-1+j, t=1-j
$$

The main advantage of the complex representation in (1) is that it conserves all the information in the initial symbolic sequence and makes evident the afore mentioned regularities of the sequences. Additionally, it is unbiased, i.e. it introduces no artifacts related to any specific assumptions on the types of interaction that characterize the nucleotides [24].

The statistics of nucleotides can be described simply by two signatures:

\section{A. The Nucleotide Imbalance}

$$
N_{c}=3\left(n_{G}-n_{C}\right)+\left(n_{A}-n_{T}\right)=(4 / \pi) \vartheta_{c}
$$

where $n_{A}, n_{C}, n_{G}$ and $n_{T}$ are the numbers of adenine, cytosine, guanine and thymine nucleotides in the sequence from the first to the current entry, and $\vartheta c$ is the cumulated phase, which is equal to the sum of the phases of all entries of the NuGS, from the beginning of the sequence, up to the current position.

\section{B. The Nucleotide Pair Imbalance}

$$
P_{u}=n_{+}-n_{-}=(2 / \pi) \vartheta_{u}
$$

where $n_{+}$is the number of positive pairs $(\mathrm{A} \rightarrow \mathrm{G}, \mathrm{G} \rightarrow \mathrm{C}, \mathrm{C} \rightarrow \mathrm{T}$, $\mathrm{T} \rightarrow \mathrm{A}$ ), and $\mathrm{n}$ - the number of negative pairs $(\mathrm{A} \rightarrow \mathrm{T}, \mathrm{T} \rightarrow \mathrm{C}$, $\mathrm{C} \rightarrow \mathrm{G}, \mathrm{G} \rightarrow \mathrm{A}$ ), and $\vartheta_{u}$ is the unwrapped phase of the entries of the $N u G S$.

\section{The Nucleotide Path}

Another useful representation of $N u G S$ is the nucleotide path $(N u P)$ of a sequence, which results due to adding the representations of the successive entries in the corresponding complex $N u G S$. The real and the imaginary part of $N u P$ give the weak-strong and purine-pyrimidine imbalances, respectively:

$$
\begin{gathered}
\operatorname{Re}\{N u P\}=n_{w}-n_{S}=\left(n_{A}+n_{T}\right)-\left(n_{G}+n_{C}\right) \\
\operatorname{Im}\{N u P\}=n_{r}-n_{y}=\left(n_{A}+n_{G}\right)-\left(n_{T}+n_{C}\right)
\end{gathered}
$$

where $n_{w}$ and $n_{s}$ are the numbers of weak and strong links, $n_{r}$ and $n_{y}$-the numbers of purine and pyrimidine, $n_{A}, n_{C}, n_{G}$ and $n_{T}$ are the numbers of adenine, cytosine, guanine and thymine nucleotides in the sequence from the start to the current position.

\section{MitochondRial DNA, NEURodEgENERATIVE AGING-RELATED GENES}

COX1 cytochrome $\mathrm{c}$ oxidase subunit $\mathrm{I}$ and $\mathrm{CYTB}$ cytochrome $b$ are two mitochondrial genes which were chosen due to their relatedness to neurodegenerative diseases and availability in database resources.

\section{A. COXI Cytochrome c Oxidase Subunit I}

MT-CO1 is the mitochondrial protein-coding gene. It has role in catalysis the reduction of oxygen to water. Cytochrome c oxidase I COX1, a homologue of MT-CO1, has been related to longevity in the filamentous fungus Podospora anserine [28]. Mutations in the human MT-CO1 gene have been associated with several diseases, including neurodegenerative diseases [29]. Further research is 
necessary to understand whether MT-CO1 is involved in human ageing. COX1 is specified in Table I and Fig. 3.

MT-CO1 is a multi-chain transmembrane protein located in the inner membrane of mitochondria and the cell membrane of prokaryotes. It catalyses the reduction of $\mathrm{O}_{2}$ and simultaneously pumps protons across the membrane [29]. It belongs to cytochrome c oxidase subunit I protein family. It is often used in DNA barcoding, because its mutation rate is often fast enough to distinguish closely related species.

TABLE I: COX1 CYTOCHROME C OXIDASE SUBUNIT I

\begin{tabular}{ll}
\hline \hline Cytogenetic band & mtDNA \\
\hline Location & $5,901 \mathrm{bp}$ to $7,518 \mathrm{bp}$ \\
\hline Gene type & protein coding \\
\hline ORF (sequence length) & $1542 \mathrm{bp}$ \\
\hline CDS (sequence length) & $513 \mathrm{aa}$ \\
\hline \hline
\end{tabular}

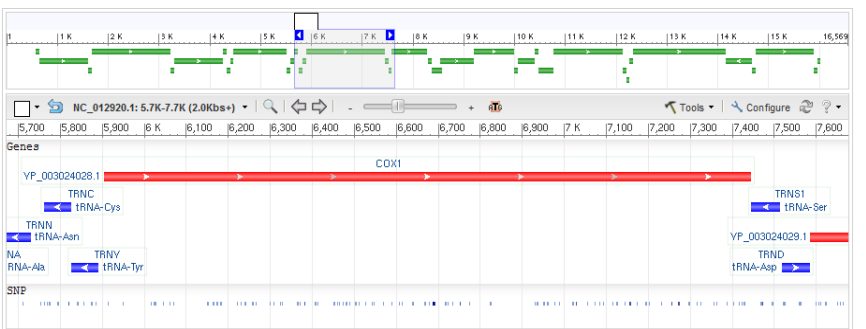

Fig. 3. Genomic regions graphics of COX1.

\section{B. CYTB Cytochrome b}

CYTB cytochrome $b$ is a mitochondrial gene, encoding protein Mt-CYB, used for population genetics and phylogenetics. Cytochrome $b$ is $a$ component of the respiratory chain complex III. These complexes are involved in electron transport and the generation of ATP and thus play $a$ vital role in the cell. Cytochrome $b$ is a subunit of cytochrome bc1, an 11-subunit mitochondrial respiratory enzyme. Cytochrome b spans the mitochondrial membrane with 8 transmembrane helices $(\mathrm{A}-\mathrm{H})$ in eukaryotes. CYTB is specified in Table II and Fig. 4.

Metabolism of energy nutrients by the mitochondrial electron transport chain (ETC) is implicated in the aging process. Polymorphisms in core ETC proteins may have an effect on longevity. Thus the cytochrome $b$ (cytb) polymorphism at amino acid 7 (cytbI7T) plays an affected role in human longevity [30].

TABLE II: CYTB CYTOCHROME B

\begin{tabular}{ll}
\hline \hline cytogenetic band & mtDNA \\
\hline Location & $14747 \mathrm{bp}$ to $15887 \mathrm{bp}$ \\
\hline Gene type & protein coding \\
\hline ORF (sequence length) & $1141 \mathrm{bp}$ \\
\hline CDS (sequence length) & $380 \mathrm{aa}$ \\
\hline \hline
\end{tabular}

Fig. 4. Genomic regions graphics of CYTB.

\section{Methodology}

Since $H$. glaber, the naked mole rat is subterranean rodent, the mitochondrial genomes of 23 species were chosen from four families: mice, rat, mole and mole-rat. And the comparison was done in the following stages:

\section{A. Multiple Sequence Alignment}

Multiple sequence alignment is used to unite the length of the species' sequences and find the similarities among them creating the phylogenetic trees.

The phylogenetic tree was created for the 23 species, and as Fig. 5 shows; H. glaber has a separate branch from mole-rat group.

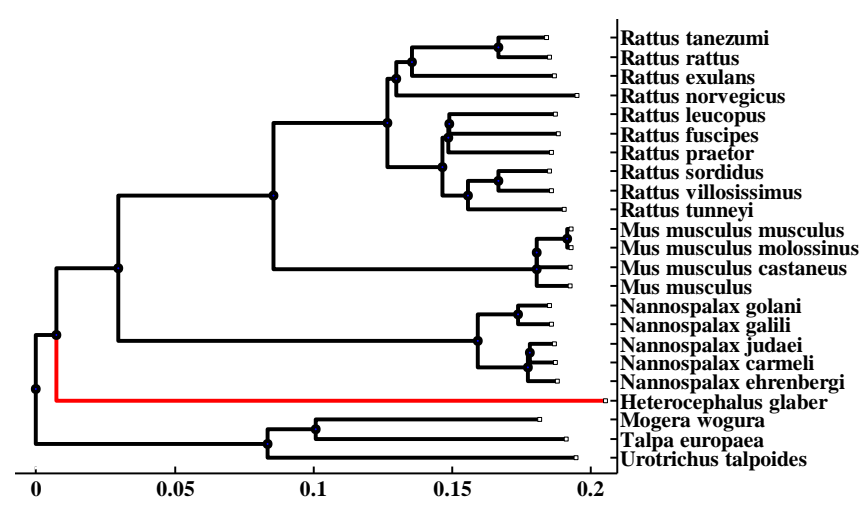

Fig. 5. Phylogenetic tree for mitochondrial genome.

\section{B. Nucleotide Genomic Signals}

The aligned mitochondrial genome sequence of each species was represented as complex number sequences. We converted it to genomic signals by applying the statistics NuGS functions, which previously described. Then we calculated the accumulated phase from the nucleotide imbalance equation (2) for the 23 species, to obtain their genomic signals and represent them. The following graph shows the Nucleotide Imbalance signals for five species.

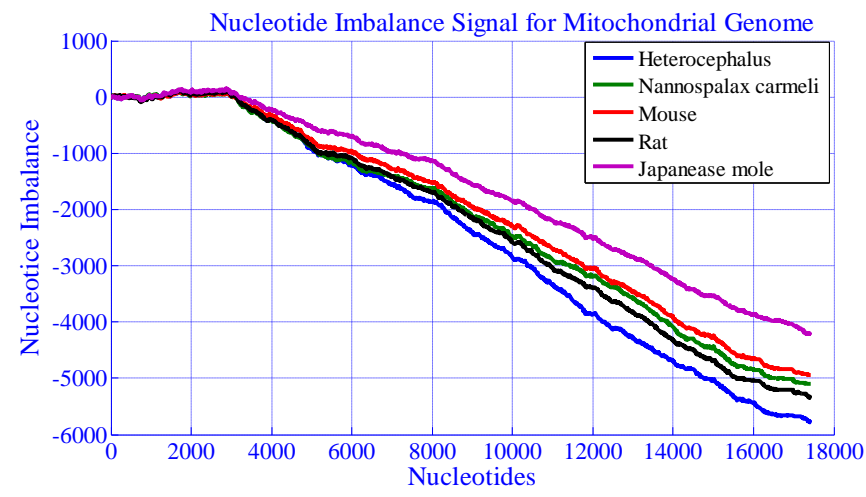

Fig. 6. The nucleotide imbalance signals for five species: Rattus rattus, Mus musculus, Nannospalax carmeli, Mogera wogura (Japanese mole) and $H$. glaber.

\section{Hierarchical Cluster and the Nearest Neighbour Calculations}

The pairwise Euclidean distance was calculated between pairs of objects (nucleotide distributions (imbalance) values of the species) in $m$-by- $n$ data matrix $X$. The distances $d$ compose a row vector of length $m(m-1) / 2$, corresponding to pairs of observations (species) in the data matrix $X$. The distances are arranged in the order $(m, m-1)$ that start from 
$m=2$. $\quad d$ is commonly used as a dissimilarity matrix in clustering or multidimensional scaling. The square Euclidean distance matrix $D$ was created by converting the $d$ distances vector to the square form. A full dissimilarity matrix must be real and symmetric, and should have zeros along the diagonal, and positive elements everywhere else. $D$ can be specified also as a full similarity matrix, with ones along the diagonal and all other elements less than one.

Clusters are based on the single linkage algorithm using Euclidean distances between the rows of $X$ matrix, which represents the nucleotide imbalance values of the genome sequence. The linkage matrix $Z$ is a $(m-1=22)$ by 3 matrix, where $m$ is the number of observations in the original data and is equal to 23 .

The dendrogram was created from the linkage matrix, it consists of many U-shaped lines connecting objects in a hierarchical tree. The height of each $U$ represents the distance between the two objects being connected.

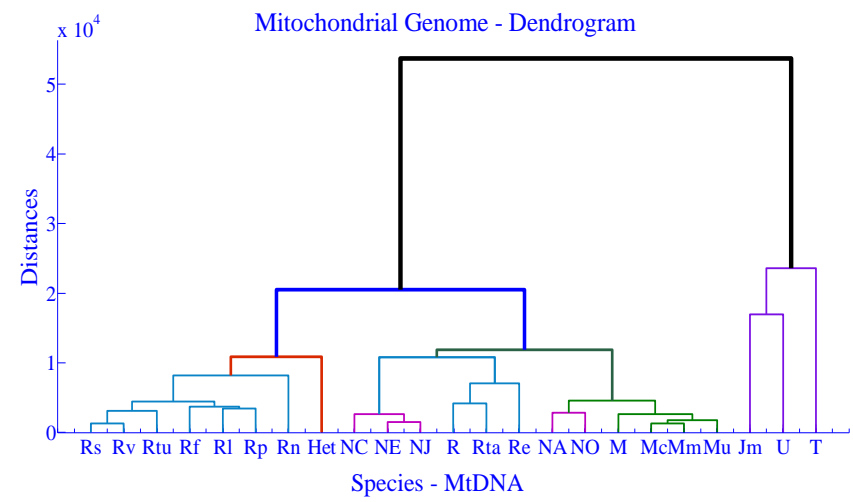

Fig. 7. Dendrogram of MtDNA of 23 species. Rs: Rattus sordidus, Rv: Rattus villosissimus, Rtu: Rattus tunneyi, Rf: Rattus fuscipes, Rl: Rattus leucopus, Rp: Rattus praetor, Rn: Rattus norvegicus, Het: Heterocephalus glaber, NC: Nannospalax carmeli, NE: Nannospalax ehrenbergi, NJ: Nannospalax judaei, R: Rattus rattus, Rta: Rattus tanezumi, Re: Rattus exulans, NA: Nannospalax galili, No: Nannospalax golani, M: Mus musculus, Mc: Mus musculus castaneus, Mm: Mus musculus molossinus, Mu: Mus musculus musculus, Jm: Mogera wogura, U: Urotrichus talpoides, T: Talpa europaea.

The distances are represented by a classical scaling configuration matrix $Y$ with dimensions $n=23$ and $p=22$. Rows of $Y$ are the coordinates of $n$ points in $p$-dimensional space. $p$ is the dimension of the smallest space in which the $n$ points whose inter-point distances are given by $D$ can be inserted.

But we can reduce the dimensions by estimating the elements of eigenvalues $e$ of $Y * Y^{\prime}$. The first $p$ elements of $e$ are positive, the rest are equal to zero. If the first $k$ elements of $e$ are much larger than the remaining $(n-k)$, then you can use the first $k$ columns of $Y$ as $k$-dimensional points whose inter-point distances approximate $D$. This can provide a useful dimension reduction for visualization, and in our experiment the first three elements are positive and much larger than the rest of elements so we can present the distances in $2 \mathrm{D}$ or $3 \mathrm{D}$.

The coordinates of two-dimensional distance map are represented from the first and second columns of the configuration matrix of the square distance matrix. The Fig. 8 shows the distance map of the mitochondrial genome of 23 species, $H$. glaber is closer to the rats cluster.

The three-dimensional map of the distances among the species is represented by using the first three columns of the configuration matrix as coordinates of the each organism in three-dimensional space.

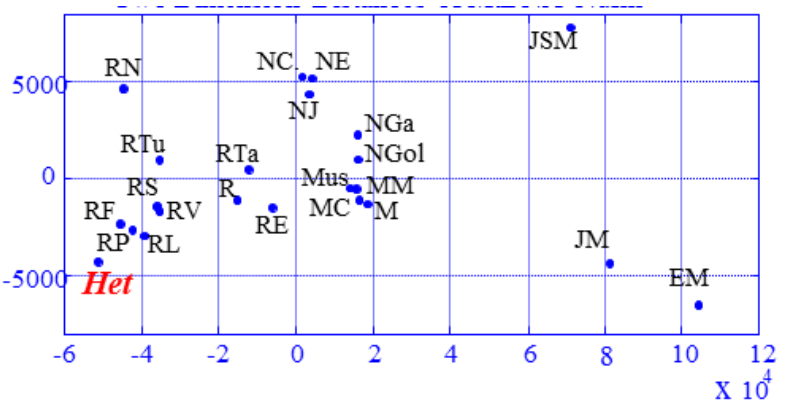

Fig. 8. Two-dimensional distance map of nucleotide imbalance of mitochondrial genome. Rs: Rattus sordidus, Rv: Rattus villosissimus, Rtu: Rattus tunneyi, Rf: Rattus fuscipes, R1: Rattus leucopus, Rp: Rattus praetor, $\mathrm{Rn}$ : Rattus norvegicus, Het: Heterocephalus glaber, NC: Nannospalax carmeli, NE: Nannospalax ehrenbergi, NJ: Nannospalax judaei, R: Rattus rattus, Rta: Rattus tanezumi, Re: Rattus exulans, NA: Nannospalax galili, No: Nannospalax golani, M: Mus musculus, Mc: Mus musculus castaneus, Mm: Mus musculus molossinus, Mu: Mus musculus musculus, Jm: Mogera wogura, U: Urotrichus talpoides, T: Talpa europaea.

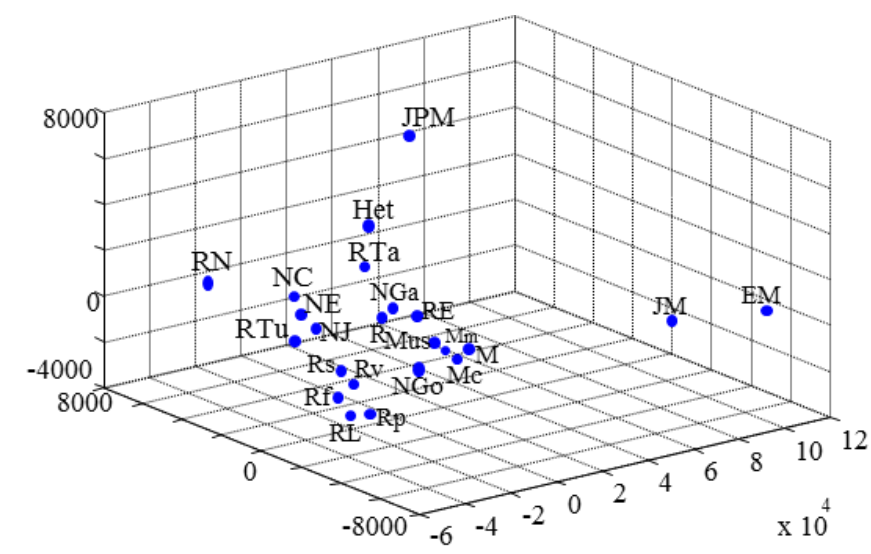

Fig. 9. Three-dimensional distance representation of nucleotide imbalance of mitochondrial genome. Rs: Rattus sordidus, Rv: Rattus villosissimus, RTu: Rattus tunneyi, Rf: Rattus fuscipes, RL: Rattus leucopus, Rp: Rattus praetor, RN: Rattus norvegicus, Het: Heterocephalus glaber, NC: Nannospalax carmeli, NE: Nannospalax ehrenbergi, NJ: Nannospalax judaei, R: Rattus rattus, RTa: Rattus tanezumi, RE: Rattus exulans, NGa: Nannospalax galili, NGo: Nannospalax golani, M: Mus musculus, Mc: Mus musculus castaneus, Mm: Mus musculus molossinus, Mus: Mus musculus musculus, JM: Mogera wogura, JSM: Urotrichus talpoides, EM: Talpa europaea.

The same procedures were applied for COX1 and CYTB gene for 8 species.

\section{CONCLUSION}

Since the mitochondrial genome of $H$. glaber is available, two aging-related mitochondrial genes were selected based on the classification of aging-related genes in Human Ageing Genomic Resources database. Cytochrome b (CYTB) and Cytochrome c oxidase subunit I (COX1) are both related to neurodegenerative disease.

The linkage was investigated by comparing the mitochondrial genomes of selected rodents close to $H$. glaber. They were selected from four genera (genuses). The calculation of the distances among the nucleotide genomic signals of the mitochondrial genome and COX1 and CYTB genes shows the following results.

The calculation of the distances for mitochondrial genome: the shortest distance to $H$. glaber is Rattus fuscipes $=1.0874$ 
$\times 10^{4}$. The calculation of the distances for CYTB gene: the shortest distance to $H$. glaber is Rattus rattus $=648.824$. The calculation of the distances for COX1 gene: the shortest distance to $H$. glaber is Rattus norvegicus $=846.769$.

\section{REFERENCES}

[1] J. P. D. Magalhaes, J. M. Sedivy, C. E. Finch, S. N. Austad, and G. M. Church, "A Proposal to sequence genomes of unique interest for research on aging," Journal of Gerontology: Biological Sciences, vol. 62A, no. 6, pp. 583-584, 2007.

[2] J. P. D. Magalhaes, "Species selection in comparative studies of aging and antiaging research," in Handbook of Models for Human Aging, Conn, pp. M. (ed.3). Elsevier Academic Press, Burlington, MA, pp. 9-20, 2006.

[3] C. T. Murphy, S. A. Carroll, C. I. Bargmann, A. Fraser, R. S. Kamath, J. Ahringer, H. Li, and C. Kenyon, "Genes that act downstream of DAF-16 to influence the lifespan of caenorhabditis elegans," Nature, vol. 424, pp. 277-283, 2003.

[4] B. Hamilton, Y. Dong, M. Shindo, W. Liu, I. Odell, G. Ruvkun, and S. S. Lee, "A systematic RNAi screen for longevity genes in C. Elegans," Genes Dev, vol. 19, pp. 1544-1555, 2005.

[5] M. Vila and S. Przedborski, "Genetic clues to the pathogenesis of parkinson's disease," Nature Medicine, Neurodegeneration, July 2004.

[6] O. Begel, J. Boulay, B. Albert, E. Dufour, and A. S. Chanet, "Mitochondrial group II introns, cytochrome c oxidase, and senescence in podospora anserina," Molecular and Cellular Biology, vol. 19, no. 6 , pp. 4093-4100, June 1999.

[7] W. Dauer and S. Przedborski, "Parkinson's disease: mechanisms and models," Neuron, vol. 39, pp. 889-909, September 2003.

[8] P. D. Cristea. "Conversion of nucleotides sequences into genomic signals," Journal of Cellular and Molecular Medicine, vol. 6, Issue 2 , pp. 279-303, May 2007.

[9] I. Provaznik, V. Kubicova, H. Skutkova, J. Nedved, E. Tkacz, P. Babula, and R. Kizek, "Detection of short exons in DNA sequences using complex wavelet transform of structural features," in Proc. of The 2012 IEEE International Workshop on Genomic Signal Processing and Statistics (GENSIPS'12), Washington, DC, USA, in press, 2012.

[10] E. Keedwell and A. Narayanan, "Intelligent Bioinformatics; The application of artificial intelligence techniques to bioinformatics problems," John Wiley and Sons Ltd, England, pp. 3-99, 2005.

[11] P. W. Sherman, J. U. M. Jarvis, and R. D. Alexander, The Biology of the Naked Mole-Rat. Princeton, NJ: Princeton University Press, 1991.

[12] J. P. de Magalhaes, "Species selection in comparative studies of aging and antiaging research," in Handbook of Models for Human Aging, P. M. Conn (ed.). Elsevier Academic Press, Burlington, MA, pp. 9-20, 2006.

[13] J. P. de Magalhaes, J. Costa, and G. M. Church, "An analysis of the relationship between metabolism, developmental schedules, and longevity using phylogenetic independent contrasts," J Gerontol A Biol Sci Med Sci, vol. 62, pp. 149-160, 2007.

[14] C. G. Faulkes, E. Verheyen, W. Verheyen, J. U. Jarvis, and N. C. Bennett, "Phylogeographical patterns of genetic divergence and speciation in African mole-rats (Family: Bathyergidae)," Mol Ecol, vol. 13 , pp. 613-629, 2004.

[15] J. L. Deuve, N. C. Bennett, J. B. Davidian, and T. J. Robinson, "Chromosomal phylogeny and evolution of the African mole-rats (Bathyergidae)," Chromosome Res, vol. 16, pp. 57-74, 2008.

[16] T. P. O'Connor, A. Lee, J. U. Jarvis, and R. Buffenstein, "Prolonged longevity in naked mole-rats: age-related changes in metabolism, body composition and gastrointestinal function," Comp Biochem Physiol A Mol Integr Physiol, vol. 133, pp. 835-842, 2002.

[17] R. Buffenstein, "Negligible senescence in the longest living rodent, the naked mole-rat: insights from a successfully aging species," J Comp Physiol B., vol. 178, no. 4, pp. 439-45, January 2008.

[18] A. Csiszar, N. Labinskyy, Z. Orosz, Z. Xiangmin, R. Buffenstein, and Z. Ungvari, "Vascular aging in the longest-living rodent, the naked mole rat," Am J Physiol Heart Circ Physiol, vol. 293, pp. 919-927, 2007.

[19] D. Huchon, O. Madsen, M. J. Sibbald, K. Ament, M. J. Stanhope, F. Catzeflis, W. W. D. Jong, and E. J. Douzery, “ Rodent phylogeny and a timescale for the evolution of Glires: evidence from an extensive taxon sampling using three nuclear genes," Mol Biol Evol, vol. 19, pp. 1053-1065, 2002.

[20] M. S. Springer, W. J. Murphy, E. Eizirik, and S. J. O. Brien, "Placental mammal diversification and the cretaceous-tertiary boundary," Proc Natl Acad Sci USA, vol. 100, pp. 1056-1061, 2003.
[21] T. B. Kirkwood and S. N. Austad, "Why do we age?" Nature, vol. 408 pp. 233-238, 2000

[22] D. Harman, “The aging process," Proc Natl Acad Sci, USA, vol. 78, pp. 7124-7128, 1981.

[23] K. B. Beckman and B. N. Ames, "The free radical theory of aging matures," Physiol Rev, vol. 78, pp. 547-581, 1998.

[24] P. D. Cristea, "Comparative analysis of mitochondrial DNA by using nucleotide genomic signals," Materials Science Forum, vol. 670, pp. 507-516, 2011.

[25] P. D. Cristea and R. Tuduce, "Nucleotide genomic signal comparative analysis of homo sapiens and other hominidae mtDNA," in Proceedings ISSCS 2009-International Symposium on Signals, Circuits and Systems, Iaşi, Romania, pp. 5-8, 2009.

[26] B. Magasanik, E. Vischer, R. Doniger, D. Elson, and E. Chargaff, "The separation and estimation of ribonucleotides in minute quantities," $J$ Biol Chem, vol. 186, no. 1, pp. 37-50, 1950.

[27] P. D. Cristea, E. Daugherty, I. Shmulevich, J. Chen, and Z. J. Wang, "Representation and analysis of DNA sequences," in Genomic Signal Processing and Statistics, Eds. EURASIP Book Series on Signal Processing and Communications, Hindawi, pp.15-65, 2005.

[28] O. Begel, J. Boulay, B. Albert, E. Dufour, and A. S. Chanet, "Mitochondrial group II introns, cytochrome c oxidase, and senescence in Podospora anserina," Mol Cell Biol, vol. 19, pp. 4093-4100, 1999.

[29] M. D. Brown, A. S. Voljavec, M. T. Lott, I. MacDonald, and D. C. Wallace, "Leber's hereditary optic neuropathy; A model for mitochondrial neurodegenerative diseases," FASEB J, vol. 6, no. 10, pp. 2791-2799, 1992.

[30] W. A. Beckstead, M. T. W. Ebbert, M. J. Rowe, and D. A. McClellan, "Evolutionary Pressure on Mitochondrial cytochrome $b$ is consistent with a role of cytbI7T affecting longevity during caloric restriction," PLoS ONE, vol. 4, no. 6, pp. 5836, 2009.

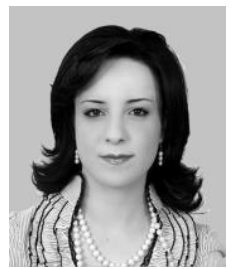

Layal Abo Khayal received the Engineer degree in biomedical engineering in 2008 from Damascus University of Technology, Syria Republic. She began her doctorate study in 2010 in Brno University of Technology, Czech Republic. She is member of IIIS IACSIT and APCBEES. She is doing her doctorate study under Prof. Ivo Provaznik's supervision. Her research interests include bioinformatics and processing of genetic and proteomic data. She has publications in impact journal and international conferences.

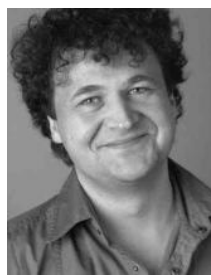

Ivo Provazník received the $\mathrm{Ph} . \mathrm{D}$. degree in biomedical engineering in 1991 from Brno University of Technology, Czech Republic. He was a visiting scientist at Johns Hopkins University, MD, USA. He is currently Professor of biomedical engineering with the Brno University of Technology and the head of Department of Biomedical Engineering. He is the author or coauthor of more than 70 scientific papers published in reviewed journals and presented at international conferences indexed in WoS. He supervises Bachelor and Master's study programs in bioinformatics. His research interests include genomics, bioinformatics and biomedical signal processing.

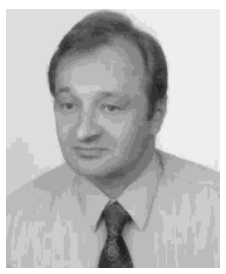

Ewaryst J. Tkacz obtained the Ph.D. degree from the Technical University of Brno, Institute of Biomedical Engineering, Czech Republic in 1987. Title of Professor he received from the President of Poland in 2007. Currently he is a Professor of Biomedical Engineering at the Faculty of Biomedical Engineering, Silesian University of Technology, Gliwice, Poland He leads there the Department of Biosensors and Biomedical Signal Processing. Also he is a Professor of BME at the Brno University of Technology, Institute of Biomedical Engineering, Czech Republic. His scientific interests are focused around various methods for biomedical signals processing, neural engineering and combination of both mentioned previously e.g. wavelet neural networks. New methods of HRV signal analysis have been elaborated during Visiting Assistant Professor stays at the Behavioral Medicine Research Center and Department of Biomedical Engineering of the University of Miami, Florida, USA. 